Revista de Investigación Cientiffica y Tecnolo̊gica

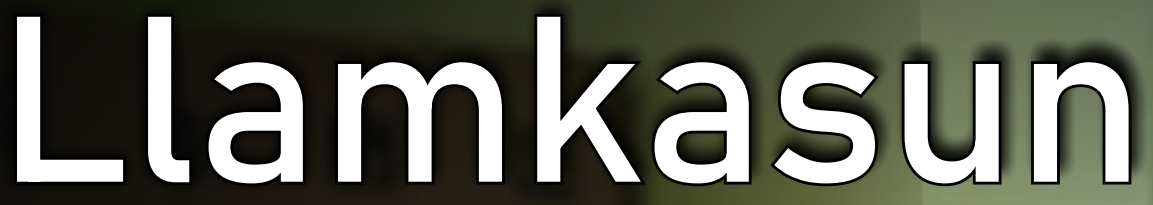

ISSN: 2709-2275

Trabajemos

Vol. 02 Num. 03 - Junio 2021

Retroalimentación asertiva en un contexto de enseñanza a distancia como producto de la COVID-19

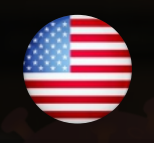

Assertive feedback in a distance learning context as

a product of COVID-19

6. Feedback Assertivo num Contexto de Ensino à Distância como produto da COVID-19

Edición Especial

COVID - 19

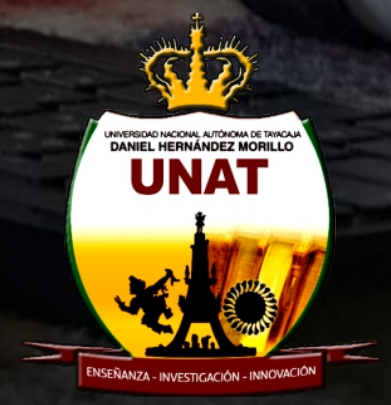

UNIVERSIDAD NACIONAL AUTÓNOMA DE TAYACAJA DANIEL HERNÁNDEZ MORILLO VICEPRESIDENCIA DE INVESTIGACIÓN 


\section{Retroalimentación asertiva en un contexto de enseñanza a distancia como producto de la COVID-19}

\section{Assertive feedback in a distance learning context as a product of COVID-19 \\ Feedback Assertivo num Contexto de Ensino à Distância como produto da COVID-19}

Elizabeth del Rosario Bringas Usquiano

Universidad César Vallejo

Mildred Jénica Ledesma Cuadros

Universidad César Vallejo

\section{RESUMEN}

El estudio tiene como objetivo determinar las diferencias que existen en el nivel de retroalimentación asertiva en el contexto de la enseñanza a distancia producida por la COVID19 en estudiantes de instituciones educativas de educación superior pedagógica de gestión pública y privada de Lima. El estudio se desarrolló bajo un enfoque cuantitativo, descriptivo comparativo, de tipo básico y con un corte transversal; la población del estudio estuvo representada por los estudiantes de las instituciones educativas superiores en mención, la muestra fue de 400 estudiantes. Los resultados arrojaron que existen diferencias significativas según los estudiantes de educación en las dimensiones que describen en la retroalimentación asertiva en una educación a distancia producida por la COVID-19 en las instituciones educativas de educación superior donde se realizó el estudio. La presente investigación respecto a la ejecución de la retroalimentación asertiva en el contexto de la enseñanza a distancia indica que no existen diferencias significativas considerando el tipo de institución y el sexo de los estudiantes, lo que finalmente significa que la gestión de la retroalimentación asertiva en el contexto de la enseñanza a distancia presenta características similares.

Palabras clave: retroalimentación, evaluación, enseñanza a distancia, COVID-19. 


\begin{abstract}
The objective of the study is to determine the differences that exist in the level of assertive feedback in the context of distance learning produced by COVID-19 in students of educational institutions of higher pedagogical education of public and private management in Lima. The study was developed under a quantitative, descriptive-comparative, basic and cross-sectional approach; the study population was represented by the students of the higher educational institutions in question, the sample was 400 students. The results showed that there are significant differences according to the students of education in the dimensions described in the assertive feedback in a distance education produced by COVID-19 in the educational institutions of higher education where the study was carried out. The present research regarding the implementation of assertive feedback in the context of distance education indicates that there are no significant differences considering the type of institution and gender of the students, which finally means that the management of assertive feedback in the context of distance education presents similar characteristics.
\end{abstract}

Keywords: feedback, evaluation, distance learning, COVID-19.

\title{
RESUMO
}

O estudo visa determinar as diferenças que existem no nível de feedback assertivo no contexto do ensino à distância produzido pela COVID-19 nos estudantes das instituições educativas de gestão pedagógica pública e privada do ensino superior de Lima. O estudo foi desenvolvido sob uma abordagem quantitativa, descritiva, comparativa, básica e transversal; a população estudada foi representada por estudantes das instituições de ensino superior em questão, a amostra foi de 400 estudantes. Os resultados mostraram que existem diferenças significativas de acordo com os estudantes de educação nas dimensões descritas no feedback assertivo num ensino à distância produzido pela COVID-19 nas instituições educacionais do ensino superior onde o estudo foi realizado. A presente investigação relativa à implementação de feedback assertivo no contexto do ensino à distância indica que não existem diferenças significativas tendo em conta o tipo de instituição e o género dos estudantes, o que finalmente significa que a gestão do feedback assertivo no contexto do ensino à distância apresenta características semelhantes.

Palavras-chave: feedback, avaliação, ensino à distância, COVID-19. 


\section{INTRODUCCIÓN}

A partir del año 2020 el mundo está viviendo, la pandemia producida por la COVID-19, la cual detuvo abruptamente las actividades a nivel mundial; provocando una alteración severa en la educación en toda la historia del mundo. De acuerdo a información de la Unesco, durante el pico más alto de la pandemia un total de 1600 millones de estudiantes de más de 190 países no asistieron a las escuelas, más de 100 millones de docentes y de personal de las instituciones educativas fueron afectadas por el cierre inesperado de éstas y todavía hoy en día dos tercios de toda la población de estudiantes en el mundo aún se encuentran sin asistir a las instituciones educativas de manera presencial por el cierre parcial o total de las instituciones educativas y 29 países permanecen totalmente cerradas (Ordorika, 2020). Ello constituye un tema preocupante para los gobiernos de los países.

La enseñanza a distancia fue una alternativa de emergencia por la coyuntura pandémica la cual consiste en una transformación temporal y repentina de las técnicas y métodos de enseñanza causada por las circunstancias propias de la crisis sanitaria, siendo su objetivo principal otorgar temporalmente acceso a los recursos y herramientas de enseñanza de una formas más rápida y factible de manejar, implementándose diversas estrategias alternativas para el aprendizaje, como el uso de celulares, radio, televisión, páginas web u otras de acuerdo a la realidad (Torres, 2020).

Por lo antes expuesto, dentro del contexto de la región latinoamericana y caribeña se ha propuesto la implementación de la enseñanza a distancia para dar solución a tan álgido problema a corto plazo, conservando la continuidad de las actividades de aprendizaje. Cada país adecua las propuestas de acuerdo a su realidad y contexto con los recursos, medios $\mathrm{y}$ herramientas con las que cuenta para brindar una propuesta pedagógica de emergencia en esta educación a distancia (Ríos et al., 2020)

A diferencia de otros países según Valentini \& González (2020) en Argentina y Perú, las autoridades del sector educación acordaron que las calificaciones de los estudiantes durante el confinamiento se realicen a través de una evaluación formativa, considerada como una medida primordial ante la emergencia sanitaria; para que los docentes afiancen e intensifiquen la autogestión del aprendizaje, siendo la 
retroalimentación clara y sistemática el camino para ello.

El contexto de aislamiento social en que se encuentra el Perú conlleva a desarrollar aprendizajes en los estudiantes de las diferentes modalidades a nivel nacional por medio de la implementación de "Aprendo en casa", como una estrategia de educación no presencial, lo que permitiría a los estudiantes el logro de sus aprendizajes tomando en cuenta sus características, necesidades, intereses y ritmos, reflexionar sobre sus fortalezas y debilidades, siendo el aspecto más importante el desarrollo de su autonomía, generándose por ello, la relevancia de la retroalimentación (Dirección Regional de Educación de Ayacucho - DREA, 2020).

\section{Revisión de la literatura de la retroalimentación asertiva}

La enseñanza a distancia de emergencia es un término que ha generado diversas opiniones en el seno de la sociedad académica, surgiendo como un término opcional común usado por investigadores. Se ha elegido el término "enseñanza" sobre otros como instrucción $\mathrm{o}$ aprendizaje, debido a que representa la esencia de la praxis docente según lo propuesto por. Esta enseñanza remota es una variabilidad provisional de brindar las actividades considerando diferentes alternativas de una manera rápida y fácil implementando pautas como la radio, aprendizaje combinado $\mathrm{u}$ otros medios que sean accesibles en el contexto (Fardoun et al., 2020).

Aquellos estudiantes que junto con sus familias se encuentran en contexto geográficos de difícil acceso, cuya conectividad de internet muchas veces es inestable y además comparten un solo dispositivo móvil, lo cual les genera estrés, sumado a ello; la coyuntura pandémica la cual provoca una gran brecha, corriéndose el riesgo de que los estudiantes obtengan resultados inadecuados. Por ello, es necesario que el gobierno anuncie que el perjuicio originado en este tiempo pueda resarcirse a través de estrategias que permitan el logro de los aprendizajes esperados, lo que significa no solo ponerse al día (Crespo, 2021).

Por su parte, Minedu (2020) definió la educación a distancia, como una forma de enseñar sin necesidad de que los estudiantes asistan a un lugar físico, ni compartan loas ambientes con sus pares y docentes, por lo que el desarrollo de los aprendizajes se ejecuta por medios de comunicación tecnológicos y escritos, 
implementando el recurso "Aprendo en casa", para asegurar el servicio educativo, indicando que el docente debe hacer seguimiento a los estudiantes a través de la retroalimentación para que puedan reajustar oportunamente sus estrategias de apoyo y que los estudiantes puedan hacer reflexión sobre sus aprendizajes.

La educación a distancia es el camino para que los temas estructurados en forma apropiada favorezcan la investigación en el estudiante, en vez de limitarse solo a la utilización de la memoria, promoviendo el desarrollo de una evaluación la cual permita la interactividad, pudiendo también recibir los resultados de manera inmediata y las opiniones por parte del profesor (Juca, 2016).

La enseñanza remota es una ocasión imprevista para potenciar el proceso innovador, pudiendo modificar la manera de impartir el conocimiento por las formas propias de la virtualidad en el futuro próximo cercano, lo que significaría el acceso de todos los estudiantes a la virtualidad (García, 2021).

Briz et al. (2020) afirmaron que los estudiantes deben involucrarse en su aprendizaje, puesto que, es importante para la retroalimentación, la cual puede darse por parte del docente al estudiante, con sus pares o también para que los estudiantes puedan ofrecerle sugerencias al profesor con respecto a las actividades que desarrolla; por ello, es conveniente preguntarles cómo advierten la enseñanza que reciben para mejorarla. De la misma manera, Osorio \& López (2014) afirmaron que la retroalimentación es el desarrollo fundamental a los procesos de enseñanza aprendizaje, del empleo del error para el apoyo en el momento preciso en el logro de un aprendizaje relevante y significativo; asimismo, indican que la retroalimentación beneficia a los estudiantes en sentirse seguros y reflexionar acerca de lo que están haciendo, pero también hacer una pausa para internalizar los resultados obtenidos y así diferenciar cuales son las actividades a ejecutar de acuerdo al contexto que se le presenta.

Por su parte, Minedu (2020) refirió que la retroalimentación responde a criterios basados en las interacciones de aprendizaje en el aula, con errores, aciertos, debilidades y fortalezas de los estudiantes. En ese sentido la DREA (2020) consideró que la retroalimentación es brindar una comunicación adecuada con la finalidad de optimizar en el camino de la enseñanza aprendizaje, detallando los 
logros, dificultades, planificando mecanismos en la mejora y ayuda al estudiante con respecto a sus respuestas, es reportar información con la finalidad de potenciar el desarrollo interno de los educandos, dando a conocer las fortalezas o debilidades aportando sugerencias en detalle y que estrategia utilizar para mejorar.

Desde la perspectiva de varios docentes, la retroalimentación con respecto a los estudiantes no es útil; pero, las investigaciones muestran claramente que la autoevaluación es imprecisa o incluso errónea en muchos casos y que los estudiantes a menudo se aburren o aprenden poco, de lo que se desprende que no importa mucho la retroalimentación de los profesores a los estudiantes, sino la retroalimentación de los estudiantes a los profesores para verificar si han alcanzado los objetivos, si los métodos utilizados fueron útiles, adecuados a los medios y si los estudiantes han comprendido el contenido (Wisniewski et al;2020).

Con respecto a la retroalimentación esta da acceso a que los niños y niñas se apoderen de su proceso de aprendizaje y al mismo tiempo puedan dar cuenta del mismo. No sólo de los temas trabajados sino de cómo están aprendiendo, indicando de cómo hacen uso de las estrategias que se utilizan.
De igual manera, los niños y niñas pueden acceder a una diversidad de instrumentos para la mejora de sus aprendizajes, asumiendo responsabilidades transformándose en agregado autónomo. Es necesario, brindarles información acerca de qué y cómo se está brindando el aprendizaje, mostrándoles ejemplos (Ministerio de Educación de Argentina, 2020).

La retroalimentación comprendida como un conversación que procede de una dirección propuestos por Sadler (2010) quien la definió como la acción mutua entre profesoras y educandos después del desarrollo de una actividad basada en criterios, las especificaciones de la actividad permiten orientarlos sobre el producto solicitado, a modo de información, de cómo se evaluará la evidencia; estas son compartidas por los docente y negociadas por los estudiantes dando paso para que ellos elaboren sus propias respuestas (Quezada \& Salinas, 2021).

En el caso de las aulas de los colegios de educación inicial el docente incluye dentro de su trabajo diario la retroalimentación de autorregulación promoviendo el control metacognitivo en los aprendizajes de la educación preescolar (Muñoz \& Santa Cruz, 2016). 
En referencia a los tipos de retroalimentación, Wisniewski et al. (2020) hicieron distinción entre la retroalimentación al saber, la correctiva y la de alta información, en el primer caso se aumenta o disminuye la frecuencia de una respuesta o comportamiento deseado y contiene información sobre la tarea, en la segunda que solo se presenta en forma de "bien o mal" y la disposición de la respuesta correcta a la tarea y la última constituida por la información descrita para la retroalimentación correctiva y que además contiene información sobre la autorregulación para monitorear la atención, las emociones o la motivación durante el proceso de aprendizaje.

Sánchez-Contreras (2019) indicaron que para Tobón la retroalimentación asertiva es el procedimiento en el que los docentes ofrecen, de manera afectuosa y respetuosa, información al estudiante acerca de sus logros de aprendizaje. Se dice que hay variedad de conceptos sobre la retroalimentación, algunos de los autores precisan como una fracción de la evaluación, conectándolo luego a la enseñanza, otros indican que es un proceso que se encuentra en conexión directa con la evaluación y también con la calificación, La retroalimentación tradicional, generalmente, no brinda resultados concluyentes que indiquen que el alumno comprenda el rumbo de la actividad de aprendizaje (Pereirasarmiento \& Enciso, 2019).

Considerando el rol del maestro, BerlangaRamírez \& Juárez-Hernández (2020) manifestaron que el enfoque socioformativo es originado en Latinoamérica y que antepone el crecimiento de la capacidad humana en las organizaciones, sociedades e instituciones de enseñanza, fundamentándose en la gestión de trabajos colaborativos $\mathrm{y}$ proyectos con el objetivo de que los diversos actores cooperen en la optimización de los indicadores de la calidad de vida. Asimismo, mencionaron la taxonomía socioformativa de Tobón el cual consta de cinco niveles de desempeño: Preformal, se refiere a plantear la problemática sin ideas principales ni oportunas; receptivas, este nivel aborda al desempeño automático en presencia de los problemas, recibiendo la información requerida; resolutiva, indica como comprende la información y empleo de aspectos sustanciales en torno a la resolución de problemas principales; autónomo, aborda los problemas en forma autónoma, es crítico reflexivo y argumenta su posición; y estratégico, referido al abordaje de estrategias innovadoras y 
complicadas haciendo frente con el uso de estas estrategias, orientadas hacia los valores (Berlanga-Ramírez \& JuárezHernández, 2020).

Es así que dichos niveles sirven de apoyo a los profesores para definir de qué manera los estudiantes afrontan y resuelven los problemas del entorno en relación a las tecnologías actuales de la información y comunicación, los que varían con respecto a las estrategias de resolución de problemas, las del dominio conceptual y las actitudes. En ese orden de ideas, la evaluación conlleva a un proceso de retroalimentación y reflexión como apoyo al desarrollo del talento y la resolución de problemas (Sánchez -Contreras, 2019).

\section{METODOLOGÍA}

El presente estudio corresponde a una metodología de carácter cuantitativo, con un diseño de tipo no experimental, descriptivo, comparativo, corte transversal Cairampoma (2015) al respecto manifestaron que los estudios no experimentales son los que se desarrollan sin la posibilidad de manipular de manera deliberada las variables de investigación.

En este estudio, la población estuvo constituida por 400 estudiantes, siendo la muestra de 200 estudiantes del instituto superior pedagógico privado Jesús el Maestro; y 200 estudiantes del instituto superior pedagógico público Monterrico, ambos ubicados en Lima.

Instrumento

Para la recolección de datos se utilizó la técnica de la encuesta a través del instrumento denominado "Diseño y validación de un instrumento para evaluar la retroalimentación asertiva en educación normal" (Berlanga-Ramírez \& JuárezHernández, 2020); trabajado en formulario Google, siendo los autores legales de este importante instrumento los investigadores Ma. de la Luz Berlanga Ramírez y Luis Gibran Juárez Hernández.

Se utilizó la medición de alfa de Cronbach permitiendo conocer el grado de fiabilidad del instrumento, para calcular estos indicadores se utilizó el paquete estadístico SPSS.

Niveles y Rangos: Bajo (28 - 45), Medio (46 - 63), alto (64 - 80).

Confiabilidad: Alfa de Cronbach 0.945.

Con referencia al análisis de los datos, se procesó teniendo en cuenta que la variable es cualitativa ordinal, siendo los niveles utilizados para su valoración: Preformal (1), Receptivo (2), Resolutivo (3), Autónomo (4) y Estratégico (5).

Este estudio tuvo en cuenta la autoría de cada una de las investigaciones 
consideradas en este trabajo, tal como lo señaló Diaz (2018) sobre la importancia que tiene considerar la propiedad intelectual de los autores.

\section{RESULTADOS}

\section{Análisis descriptivo}

Como se observa en la tabla 1 y figura 1 , la ejecución de la retroalimentación asertiva en el contexto de la enseñanza remota en las instituciones evaluadas, resultó Preformal en un $1 \%$, Receptivo en un $10 \%$, Resolutivo en un 26\%, Autónomo en un $32.5 \%$ y Estratégico en un $30.5 \%$.

\section{Tabla 1}

Ejecución de la retroalimentación asertiva en el contexto de la enseñanza remota.

\begin{tabular}{lcc}
\hline \multicolumn{1}{c}{ Nivel } & Frecuencia & Porcentaje \\
\hline Preformal & 2 & 1,0 \\
Receptivo & 20 & 10,0 \\
Resolutivo & 52 & 26,0 \\
Autónomo & 65 & 32,5 \\
Estratégico & 61 & 30,5 \\
Total & 200 & 100,0 \\
\hline Fuente: Base & de datos cuestionario de & de \\
Retroalimentación Asertiva SPSS V.25 &
\end{tabular}

\section{Figura 1}

Diagrama de frecuencias de la ejecución de la retroalimentación asertiva en el contexto de la enseñanza remota



Como se observa en la figura 2 , en la ejecución de la retroalimentación asertiva en el contexto de la enseñanza remota en las instituciones evaluadas, en las mujeres prevalece el nivel autónomo con un $32.7 \%$ en comparación con los hombres en donde prevalece el nivel resolutivo con un $50 \%$. 


\section{Figura 2}

Diagrama de frecuencias de la ejecución de la retroalimentación asertiva en el contexto de la enseñanza remota según sexo



Como se observa en la tabla 2 y figura 3 , instituciones educativas públicas con un en la ejecución de la retroalimentación $33.3 \%$ en comparación con las privadas en asertiva en el contexto de la enseñanza donde prevalece el nivel estratégico con remota en las instituciones evaluadas un $33.7 \%$.

prevalece el nivel autónomo en las

\section{Tabla 2}

Ejecución de la retroalimentación asertiva en el contexto de la enseñanza remota según tipo de institución

\begin{tabular}{cccc}
\hline & & \multicolumn{2}{c}{ Tipo de gestión de la Institución } \\
\cline { 3 - 4 } Ejecución de la & Preformal & 2 & Privada \\
retroalimentación & & $1.9 \%$ & 0 \\
& Receptivo & 12 & $0.0 \%$ \\
& & $11.1 \%$ & $8.7 \%$ \\
& Resolutivo & 28 & 24 \\
& & $25.9 \%$ & $26.1 \%$ \\
& Autónomo & 36 & 29 \\
& & $33.3 \%$ & $31.5 \%$ \\
Estratégico & 30 & 31 \\
& & $27.8 \%$ & $33.7 \%$ \\
& & 108 & 92 \\
& & $100.0 \%$ & $100.0 \%$ \\
\hline
\end{tabular}

Fuente: Base de datos cuestionario de Retroalimentación Asertiva SPSS V.25 


\section{Figura 3}

Diagrama de frecuencias de la ejecución de la retroalimentación asertiva en el contexto de la enseñanza remota según tipo de institución

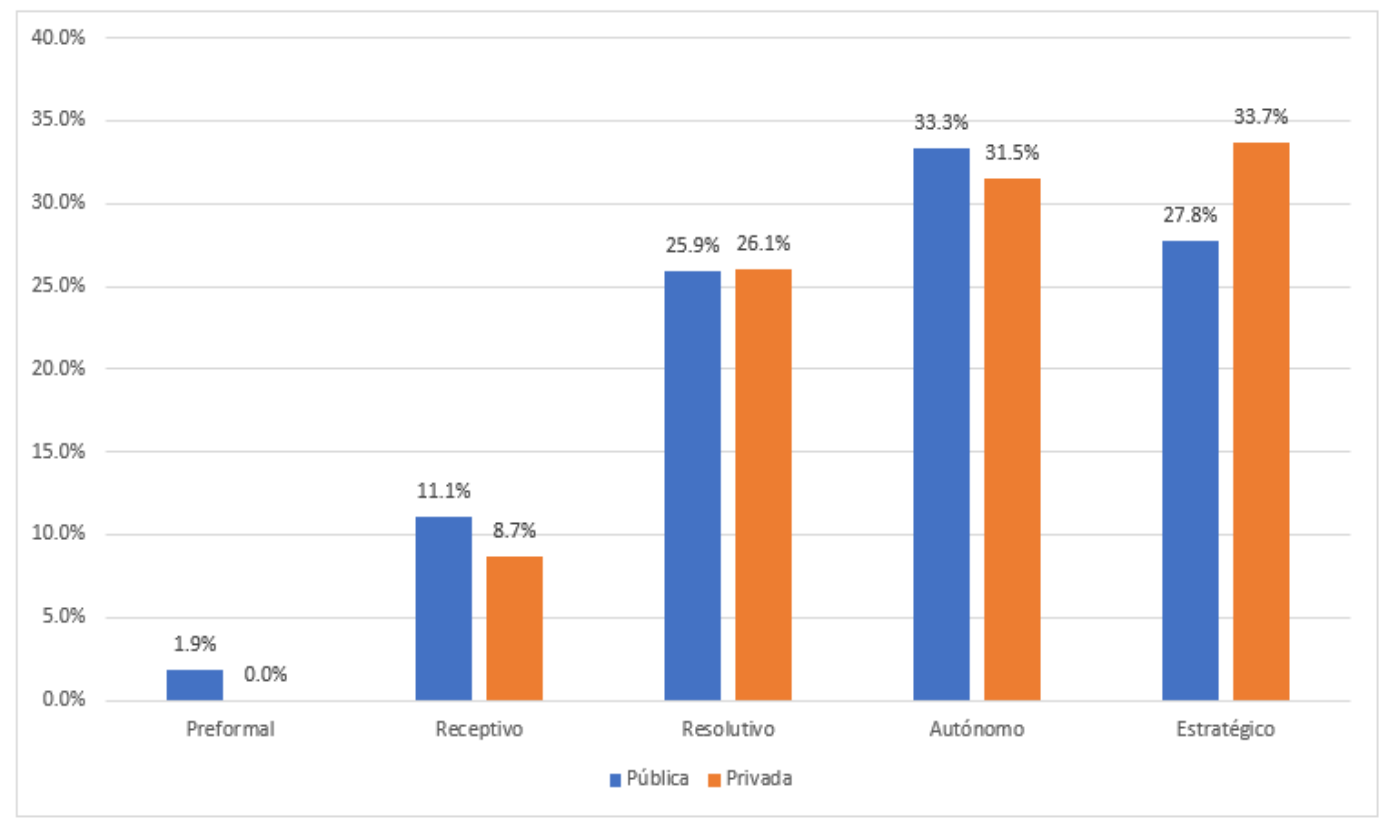

\section{Análisis Inferencial}

\section{Prueba de Hipótesis}

HE1: Existen diferencias significativas en la ejecución de la retroalimentación asertiva en el contexto de la enseñanza remota según sexo.

Ho: No existen diferencias significativas en la ejecución de la retroalimentación asertiva en el contexto de la enseñanza remota según sexo.

Regla de decisión;

Si Valor $\mathrm{p} \geq 0.05$, no se rechaza la Hipótesis Nula (Ho)

Si Valor $\mathrm{p}<0.05$, se rechaza la Hipótesis Nula (Ho). Y, se acepta Ha

En la Tabla 3, el resultado del $p_{-}$valor de la prueba de U de Mann-Whitney $p_{\text {_valor }}=$
0.091> 0.05; por tanto, no se rechazó la Ho. Por lo que se puede afirmar que: No existen diferencias significativas en la ejecución de la retroalimentación asertiva en el contexto de la enseñanza remota según sexo.

Tabla 3

Prueba de U de Mann-Whitney

Estadísticos de prueba ${ }^{a}$

Ejecución de la retroalimentación

U de Mann-Whitney 206,500

W de Wilcoxon

216,500

Z

Sig. asintótica(bilateral)

, 091

a. Variable de agrupación: Sexo

Fuente: Base de datos cuestionario de Retroalimentación Asertiva SPSS V.25 
HE2: Existen diferencias significativas en la ejecución de la retroalimentación asertiva en el contexto de la enseñanza remota según tipo de institución.

Ho: No existen diferencias significativas en la ejecución de la retroalimentación asertiva en el contexto de la enseñanza remota según tipo de institución

Regla de decisión;

Si Valor $\mathrm{p} \geq 0.05$, no se rechaza la Hipótesis Nula (Ho)

Si Valor $\mathrm{p}<0.05$, se rechaza la Hipótesis Nula (Ho). Y, se acepta Ha


prueba de $\mathrm{U}$ de Mann-Whitney $p_{\text {_valor }}=$ 0.317 > 0.05; por tanto, no se rechazó la Ho. Por lo que se puede afirmar que: No existen diferencias significativas en la ejecución de la retroalimentación asertiva en el contexto de la enseñanza remota según tipo de institución.

Tabla 4

Prueba de U de Mann-Whitney

Estadísticos de prueba

\begin{tabular}{lc}
\hline & $\begin{array}{c}\text { Ejecución de la } \\
\text { retroalimentación }\end{array}$ \\
\hline U de Mann-Whitney & 4577,000 \\
W de Wilcoxon & 10463,000 \\
$\mathrm{Z}$ & $-1,000$ \\
Sig. asintótica(bilateral) &, 317 \\
\hline
\end{tabular}

a. Variable de agrupación: Tipo de gestión de la Institución

Fuente: Base de datos cuestionario de

Retroalimentación Asertiva SPSS V.25

\section{DISCUSIÓN}

La presente investigación respecto a la ejecución de la retroalimentación asertiva en el contexto de la enseñanza remota indica que no existen diferencias significativas considerando el tipo de institución y el sexo de los estudiantes, esto validado por la prueba estadística de U de Mann-Whitney en donde las significancia bilaterales encontradas (p_valor) resultaron mayores que $0.05 ;$ lo que finalmente está significando que para la realidad geográfica estudiada, la gestión de la retroalimentación asertiva en el contexto de la enseñanza remota presenta características similares, Osorio \& López (2014) en la investigación realizada sobre retroalimentación corroboró la importancia que esta tiene en el proceso de enseñanza aprendizaje de los estudiantes. Igualmente, se puede evidenciar que la ejecución de la retroalimentación asertiva en el contexto de la enseñanza remota en las instituciones evaluadas en las mujeres prevaleció el nivel autónomo con un 32.7\% en comparación con los hombres en donde prevaleció el nivel resolutivo con un 50\%; ello puede explicarse, debido a que para el contexto y realidad estudiada a que las estudiantes mujeres tienden a madurar más rápido; por lo tanto, la capacidad crítica 
conlleva a tomar decisiones de manera autónoma.

Por otro lado, se observó que la ejecución de la retroalimentación asertiva en el contexto de la enseñanza remota en las instituciones evaluadas prevalece el nivel autónomo en las públicas con un $33.3 \%$ en comparación con las privadas en donde prevalece el nivel estratégico con un $33.7 \%$. Igualmente, descriptivamente se encontró que la ejecución de la retroalimentación asertiva según sexo en el contexto de la enseñanza remota en todas las instituciones evaluadas en el contexto de la enseñanza remota resultó Preformal en un 1\%, Receptivo en un $10 \%$, Resolutivo en un 26\%, Autónomo en un $32.5 \%$ y Estratégico en un 30.5\%; ello puede en cierta manera, explicarse debido a la implementación de la evaluación formativa en el nivel superior.

\section{CONCLUSIONES}

La investigación evidencia que no existen diferencias significativas en la ejecución de la retroalimentación asertiva en el contexto de la enseñanza remota según tipo de institución y sexo; encontrando igualmente que en las mujeres prevaleció el nivel autónomo con un $32.7 \%$ en comparación con los hombres en donde prevaleció el nivel resolutivo con un $50 \%$.
Se infiere igualmente, que estos resultados pueden estar explicados debido al contexto vivido de emergencia sanitaria y realidad geográfica donde se realizó el estudio ya que, en los institutos superiores pedagógicos, a nivel nacional se han tenido que implementar estrategias que responde a una evaluación formativa siendo la retroalimentación asertiva necesaria para que los estudiantes de formación inicial docente sean constructores de sus aprendizajes de manera autónoma y reflexiva.

\section{REFERENCIAS BIBLIOGRÁFICAS}

Berlanga-Ramírez, M. de la L., \& Juárez-Hernández， L. G. (2020). Diseño y validación de un instrumento para evaluar la retroalimentación asertiva en educación normal. IE Revista De Investigación Educativa De La REDIECH, $11, \quad \mathrm{e} 791$. https://doi.org/10.33010/ie_rie_re diech.v11i0.791

Briz, O., Escamilla, J., Galván, N., García, A., García, R., Garrido, M., González, A., Infanzón, H., Luna, V., Moreno, C., Osoria, J., Peña, A., Rivero, C., \& Rodríguez, J. 
(2020). ¿Cómo abordar la dimensión socioafectiva en la enseñanza remota de emergencia? Revista Digital Universitaria, $21(5)$ $1-10$. http://doi.org/10.22201/cuaieed.16 076079e.2020.21.5.10

Cairampoma, M. R. (2015). Tipos de Investigación científica: Una simplificación de la complicada incoherente nomenclatura y clasificación. Redvet. Revista electrónica de veterinaria, $16(1), \quad$ 1-14. http://doi.org/10.26820/recimu ndo/4.(3).julio.2020.163-173

Crespo-Antepara,

(2021). Enseñanza remota emergente. Polo del

Conocimiento, 6 (6), $\quad$ 10401051.

http://doi.org/10.23857/pc.v6i6 .2802

DREA (2020). Orientaciones para la retroalimentación en un contexto de educación a distancia.-nivel secundaria. Recuperado de https://webmaterialesdidacticos.files.w ordpress.com/2020/06/retroalimentaci on-en-la-educacion-a-distancia2020.pdf
Diaz, J. (2018). Políticas públicas en propiedad intelectual escrita. Una escala de medición para educación superior del Perú. Revista Venezolana de Gerencia, 23(81), 88-105. https://produccioncientificaluz.org/ind ex.php/rvg/article/view/23470/23679

Fardoun, H. M., González, C. S., Collazos Ordóñez, C. A., \& Yousef, M. (2020). Estudio exploratorio en Iberoamérica sobre procesos de enseñanzaaprendizaje y propuesta de evaluación en tiempos de pandemia. Education in the knowledge society, 2(1), 1-9 https://doi.org/10.14201/eks.23537

García, L. (2021). COVID-19 y educación a distancia digital: preconfinamiento, confinamiento y posconfinamiento. RIED. Revista Iberoamericana de Educación a Distancia, 24(1), 09-32. http: //dx.doi.org / 10.5944 / ried.24.1.28080

Juca Maldonado, F. J. (2016). La educación a distancia, una necesidad para la formación de los profesionales. Revista Universidad. $8(1)$. 106-111. http://scielo.sld.cu/pdf/rus/v8n1/rus 15116.pdf

Minedu (2020). Diario oficial No 15346. Recuperado de 
https://busquedas.elperuano.pe/dow

nload/full/4iOSEX-

hqYr8sqVcMUA2on

Ministerio de Educación de Argentina (2020). Retroalimentación formativa.

Recuperado de

https://radiodelmar.com.ar/wp-

content/uploads/2020/04/Retroaliment ación-en-el-marco-de-la-Evaluación-

Formativa-1.pdf

Muñoz, L, \& Santa Cruz, J. (2016). El aula preescolar como espacio de desarrollo cognitivo: tipo de feedback docente y control metacognitivo en los niños. Revista electrónica de investigación psicoeducativa, 14(38), 23-44. http://dx.doi.org/10.14204/ejrep.38. 15033

Ordorika, I. (2020). Pandemia y educación superior. Revista de la educación superior, $\quad 49(194), \quad$ 1-8. 2020.https://doi.org/10.36857/resu.202 0.194 .1120

Osorio, K., \& López, A. (2014) La Retroalimentación Formativa en el Proceso de Enseñanza-Aprendizaje de Estudiantes en Edad Preescolar. Formative Feedback in the Teaching-Learning Process of Preescolar Students. Revista
Iberoamericana de Evaluación
Educativa, $\quad 7(1), \quad 13-30$
https://revistas.uam.es/index.php/rie e/article/view/3383.

Pereira-Sarmiento, J., \& Enciso, L. (2019, June). Virtual Tutoring System with Interactive Feedback between TeacherStudent. In 2019 14th Iberian Conference on Information Systems and Technologies (CISTI) (pp. 1-6). IEEE. http://doi.org/10.23919 / CISTI.2019.8760823

Quezada, S., \& Salinas, C. (2021). Modelo de retroalimentación para el aprendizaje. Investigación, 26 (88) 225-251.

http://www.scielo.org.mx/pdf/rmie/v2 6n88/1405-6666-rmie-26-88-225.pdf

Ríos, A. M. F., Svetlichich, M., \& Durán, M.E. D. (2020). Desafíos intra-covid en américa latina para la continuidad de la educación virtual. Revista Facultad de Ciencias Contables Económicas y AdministrativasFACCEA, 10(2), $127-143$ https://doi.org/10.47847/faccea.v10 n2a1

Sadler, R. (2010). Beyond feedback: Developing student capability in complex appraisal. Assessment \& Evaluation in Higher Education, 35, 
535-550. http://doi.org/

$10.1080 / 02602930903541015$

Sánchez-Contreras, M. L. (2019).

Socioformative Taxonomy: A

referent for Didactics and

Evaluation. Forhum International.

Journal of Social Sciences and

Humanities, 1(1), 100-115.

http://dx.doi.org/10.35766/

jf19119

Torres, D. (10 de abril del 2020). En qué consiste la Enseñanza Remota de Emergencia (ERT). Estudio Torres Burriel.

https://www.torresburriel.com/weblog/ 2020/04/10/en-que-consiste-laensenanza-remota-en-emergenciasere/.

Valentini, M. y Gonzales, A. (9 de junio de 2020). Miradas latinoamericanas sobre educación en tiempos de pandemia. Reflexiones desde Argentina y Perú. Magisterio. https://www.magisnet.com/2020/06/m iradas-latinoamericanas-sobreeducacion-en-tiempos-de-pandemiareflexiones-desde-argentina-y-peru/.

Wisniewski, B., Zierer, K., \& Hattie, J. (2020). The power of feedback revisited: a meta-analysis of educational feedback research. Frontiers in

Psychology, 10, 1-

14. https://doi.org/10.3389/fpsyg.20

19.03087

\section{Contacto}

Elizabeth del Rosario Bringas Usquiano ely111963@gmail.com 